Exp Brain Res (1987) 69: 53-59

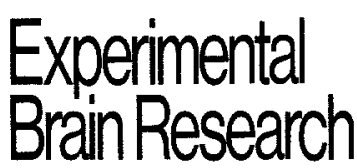

(c) Springer-Verlag 1987

\title{
Evidence for reactive synaptogenesis in the ventrolateral thalamus and red nucleus of the rat: changes in high affinity glutamate uptake and numbers of corticofugal fiber terminals
}

\author{
M. B. Bromberg, G. Pamel, B.S. Stephenson, A. B. Young, and J. B. Penney \\ Department of Neurology, University of Michigan Medical Center, 1920/0316 Taubman Center, 1500 East Medical Center Drive, \\ Ann Arbor, MI 48109-0316, USA
}

\begin{abstract}
Summary. High affinity glutamate uptake into corticofugal fiber terminals was measured in the ventrolateral thalamus and red nucleus at varying time intervals after lesions were made by kainic acid in the contralateral interpositus nucleus of the cerebellum in rats. Under similar conditions the density of cortical fiber terminals was estimated using the FinkHeimer impregnation technique. 1. Glutamate uptake steadily increased in the ventrolateral thalamus up to 60 days after lesions in the contralateral cerebellum. 2. Similar changes were noted in the red nucleus. 3 . The changes were dependent on the integrity of corticofugal fibers to the thalamus and red nucleus. 4. No changes in uptake of gammaaminobutyric acid were noted. 5. Saturation curves for glutamate uptake suggested a change in the maximal number of transport sites. 6. Fink-Heimer degeneration studies showed an increase in cortical terminals in the ipsilateral ventrolateral thalamus and in both rostral and caudal regions of the red nucleus following lesions in the contralateral interpositus nucleus. The data are consistent with an increase in the number of cortical fiber terminals in reaction to loss of cerebellar input to the ventrolateral thalamus and red nucleus. This study correlates anatomical and biochemical evidence for collateral sprouting in a model based on electrophysiologic data in the red nucleus and extends the model to include the thalamus.
\end{abstract}

Key words: Neuroplasticity - Glutamate uptake Ventrolateral thalamus - Red nucleus - Cerebellar lesion

\section{Introduction}

The creation of new synaptic connections by central nervous system neurons in response to injury has

Offprint requests to: M.B. Bromberg (address see above) been documented at a number of sites (Steward 1982 for review). Initial studies were concerned with the anatomical and electrophysiological demonstration of reactive synaptogenesis. Changes in neurotransmitter function in relation to synaptogenesis, however, have only recently been described (Pamel et al. 1981; Nieoullon et al. 1981, 1984). Although the role of reactive synaptogenesis in the compensatory process following central nervous system injury is not yet clear, information on associated changes in both preand postsynaptic neurotransmitter function is necessary before understanding any pharmacological intervention in these processes.

One of the best described models of reactive synaptogenesis occurs in the red nucleus of the cat after lesions of the interpositus nucleus of the cerebellum (Tsukahara 1978). The red nucleus receives major excitatory inputs from two principal sources, the contralateral interpositus nucleus and the ipsilateral cerebral cortex. Fibers from interpositus neurons make synaptic contact on the soma and proximal dendrites of rubral neurons (Nakamura and Mizuno 1971). The proximal synaptic location is responsible for the short rising phase of excitatory postsynaptic potentials (EPSPs) evoked by interpositus stimulation (Tsukahara et al. 1975). Cortical fibers make synaptic connections on distal dendrites of rubral neurons (Nakamura 1974; Tsukahara et al. 1975) and there is a corresponding slower EPSP rising phase after stimulation of the cortex. Following lesions of the interpositus nucleus, synaptic rearrangements of corticorubral fibers have been demonstrated both electrophysiologically and anatomically (Nakamura et al. 1974; Murakami et al. 1982). New corticorubral terminals appeared on the soma and proximal dendrites and the rise time of the cortically evoked EPSPs became shorter (Tsukahara et al. 1975). These changes occurred over several weeks after destruction of the interpositus nucleus. 
Glutamic acid has been implicated as a neuroransmitter of cortical fibers to the red nucleus in rats, cats, and monkeys (Bromberg et al. 1982; Young et al. 1981, 1983). This was demonstrated by a decrease in high affinity glutamate uptake after destruction of the corticorubral pathway. In addition, glutamate is a likely neurotransmitter in other corticofugal pathways, including those to the ventrolateral nucleus of the thalamus (Bromberg et al. 1981; Young et al. 1981, 1983; Kerkerian et al. 1982, 1983). The ventrolateral nucleus, like the red nucleus, receives fibers from the contralateral interpositus nucleus (Cajal 1911). Because of the similarities between connections to the red nucleus and portions of the thalamus, reactive synaptogenesis of corticothalamic fibers also may be expected following interpositus lesions. Supporting data have been recently presented in the rat (Nieoullon et al. 1984). In this study we investigated the time course of reactive synaptogenesis by measuring changes in high affinity glutamate uptake in corticorubral and corticothalamic fiber terminals in rats following lesions in the interpositus nucleus. Additionally, we report anatomical evidence for an increased number of cortical fiber terminals under conditions of synaptogenesis. Preliminary data have been presented (Pamel et al. 1981).

\section{Methods}

The following experimental procedures were carried out: 1) anatomical studies of fiber degeneration (Fink-Heimer) demonstrating changes in the distribution of cortical fibers to the red nucleus and ventrolateral nucleus consequent to interpositus nucleus lesions; 2) biochemical studies demonstrating the time course of changes in glutamate uptake in synaptosomes of rubral and thalamic tissue following lesions of the interpositus nucleus.

Adult Sprague-Dawley male rats $(150 \mathrm{~g})$ were anesthetized with $1.0 \mathrm{mg} / \mathrm{kg}$ of xylazine and $0.4 \mathrm{mg} / \mathrm{kg}$ ketamine and placed in a David Kopf small animal stereotaxic device with the incisor bar $5 \mathrm{~mm}$ above the intraaural line. Kainic acid was used to make lesions in the interpositus nucleus rather than fulguration because kainic acid does not destroy fibers of passage (McGeer and McGeer 1978). Lesions of the interpositus nucleus were made by iontophoretic application of $0.1 \mathrm{M}$ kainic acid dissolved in $0.01 \mathrm{M}$ Tris adjusted to $\mathrm{pH} 7.0$ with $\mathrm{HCl}$. Pulse amplitude was $1 \mathrm{mV}$ and sequence was $500 \mathrm{~ms}$ on $/ 500 \mathrm{~ms}$ off for $10 \mathrm{~min}$ and the pipette remained in place for $5 \mathrm{~min}$ after iontophoresis. At no time was seizure activity seen.

Cerebral decortication was performed in anesthetized animais whose skulls were removed bilaterally from $4 \mathrm{~mm}$ anterior to $2 \mathrm{~mm}$ posterior to bregma and from $1 \mathrm{~mm}$ to $5 \mathrm{~mm}$ lateral to bregma. The cerebral cortex was aspirated to the underlying white matter. The lesions were then extended laterally by undercutting the cortex with a curved needle for 3 to $5 \mathrm{~mm}$ along the lateral edge of the aspiration site.

Animais prepared for Fink-Heimer degeneration studies were anesthetized and perfused intravascularly with a solution of $0.4 \%$ paraformaldehyde and $1 \%$ glutaraldehyde in phosphate buffered saline at $\mathrm{pH}$ 7.4. The brains were removed from the animals, placed in a solution of $4 \%$ formaldehyde containing $30 \%$ sucrose

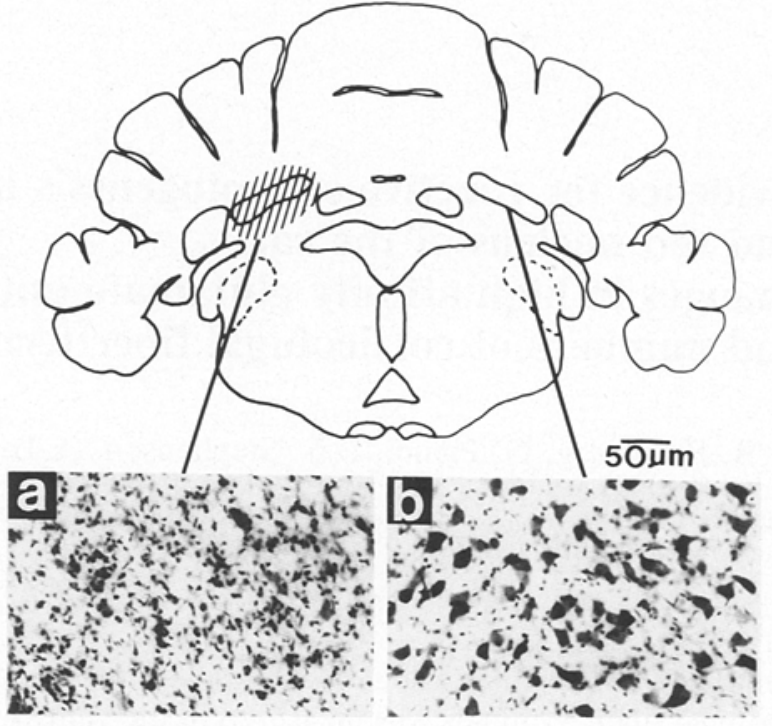

Fig. 1a, b. Lesions in the interpositus nucleus of the rat by kainic acid injection. Line drawing: Coronal section of cerebellum, hatched area in left interpositus nucleus denotes extent of lesion. a Micrograph showing cell destruction at lesion site four days after lesion. $\mathbf{b}$ Micrograph showing cytoarchitecture of intact nucleus Cresyl violet stain

for 4 days and then cut into $25 \mu \mathrm{m}$ frozen sections in the horizontal plane. The sections were stained for degenerating fibers and terminals by Fink and Heimer's second method (Fink and Heimer 1967). The prepared sections were examined in random order for the distribution of degenerating fibers and terminals by one of us (JBP) who was blind to the placement of lesions.

Animals prepared for transmitter high affinity uptake studies were decapitated, the brains rapidly removed, placed in ice cold $0.32 \mathrm{M}$ sucrose and $5 \mathrm{mM}$ sodium-potassium phosphate buffer at $\mathrm{pH} 7.4$, and cut into $1 \mathrm{~mm}$ slices at $4^{\circ} \mathrm{C}$. Small samples of tissue were obtained by pressing $1 \mathrm{~mm}$ diameter tubing through the red nucleus and ventrolateral nucleus on each side of the tissue slices and specimens were placed in $1 \mathrm{ml}$ of ice cold $0.32 \mathrm{M}$ buffered sucrose. The punch samples were homogenized in 20 to 40 volumes of ice cold $0.32 \mathrm{M}$ buffered sucrose using a Potter Elvehjem glass homogenizer fitted with a Teflon pestle. The homogenate was centrifuged at $20,000 \mathrm{~g}$ for $20 \mathrm{~min}$. The pellet was resuspended in ice cold $0.32 \mathrm{mM}$ buffered sucrose. Aliquots of the crude synaptosomal suspension were added in triplicate to $0.8 \mathrm{ml}$ of $10 \mathrm{mM}$ phosphate buffered saline containing $137 \mathrm{mM} \mathrm{NaCl}$, $1.7 \mathrm{mM} \mathrm{KCl}, 1 \mathrm{mM} \mathrm{CaCl}, 0.5 \mathrm{mM} \mathrm{MgCl}_{2}$ and $11 \mathrm{mM}$ glucose at $\mathrm{pH} 7.4$ at $4^{\circ} \mathrm{C}$. A uniform trace amount of tritiated glutamic acid or gamma-aminobutyric acid (GABA) $(10 \mathrm{Ci} / \mathrm{mmol})$ was added to nonradioactive amino acid in the incubation medium to achieve a final concentration of $1 \times 10^{-8} \mathrm{M}$. Triplicate samples were incubated for $5 \mathrm{~min}$ at $37^{\circ} \mathrm{C}$, returned to ice and centrifuged at $50,000 \mathrm{~g}$ for $10 \mathrm{~min}$ at $4^{\circ} \mathrm{C}$. Triplicate samples serving as control blanks were run in parallel but remained at $4^{\circ} \mathrm{C}$. The pellets were washed once with ice cold isotonic saline, centrifuged at $50,000 \mathrm{~g}$ for another $10 \mathrm{~min}$, and were extracted in $0.5 \mathrm{mlNCS}$ (Amersham/ Searle Corp). Six ml OCS (Amersham/Searle Corp) was added before radioactivity was determined by liquid scintillation spectroscopy.

Initial studies of uptake showed linearity of uptake with incubation time (up to $5 \mathrm{~min}$ ) and with protein concentration (up to 10 times our concentrations). In one group of animals, the high 


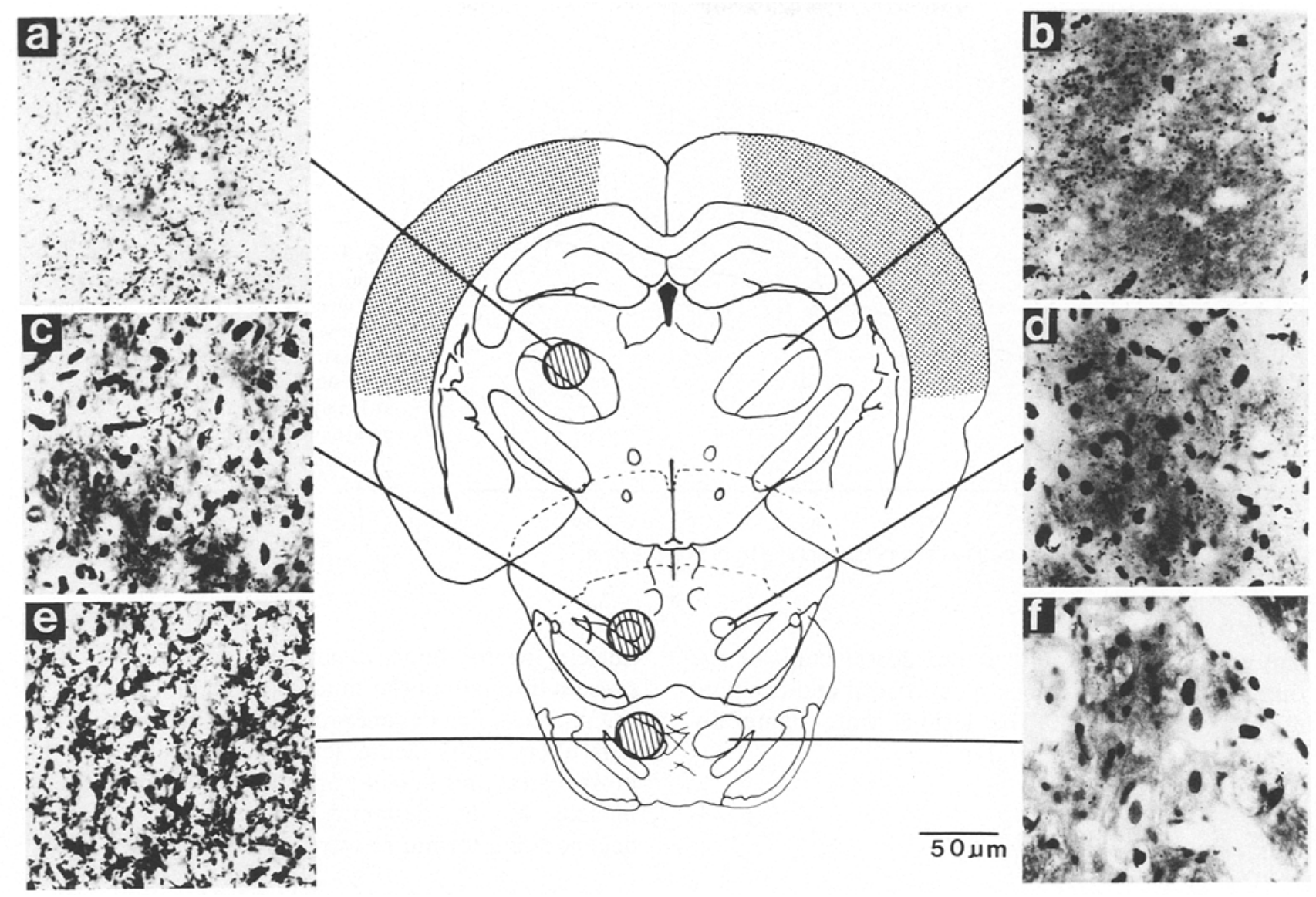

Fig. 2 a-f. Fink-Heimer degeneration study of corticofugal fibers in the rat before and after lesions of the interpositus nucleus. Line drawing: Coronal section showing extent of bilateral cortical ablation (hatched area) and ventrolateral thalamic nuclei (upper), rostral (middle) and caudal (lower) regions of red nuclei. a, c, and e Micrographs showing increased number of degenerating corticofugal fibers consequent to lesion of the contralateral interpositus nucleus 35 days earlier and 5 days after cortical ablation. $\mathbf{b}$, $\mathbf{d}$, and $\mathbf{f}$ Micrographs showing degenerating corticofugal fibers 5 days after cortical ablation only

affinity uptake of GABA was measured. In another group of animals, tissue from several ventrolateral nuclei were pooled and used to determine the saturation curve for high affinity uptake of glutamic acid. Aliquots of tissue were added in triplicate to glutamic acid concentrations varying from $1 \times 10^{-8} \mathrm{M}$ to $1 \times$ $10^{-3} \mathrm{M}$. Numbers and affinities of uptake sites were calculated according to Scatchard by computer analysis.

In all studies, except where noted, changes in the thalamic and red nuclei were compared to the opposite, unaffected nuclei which served as controls. Statistical analyses were conducted on the differenes between the affected and control sides.

All brain tissue, including the remains of the sampled midbrain and thalamic slices, sites of kainic acid interpositus nucleus lesions, and areas of decortication were placed in phosphate buffered $4 \%$ formaldehyde sucrose and fixed for one week. Fifty micron frozen sections were cut and stained with cresyl violet to ascertain accuracy and extent of lesions and punches. This examination was done by one of us (JBP) who was blind to the results of the biochemical studies. All animals in which the cerebellar lesion was not confined to the deep nuclei or in which less than $50 \%$ of the interpositus nucleus was damaged were excluded from analysis. All animals in which punches were not at least $70 \%$ within the appropriate nuclei or did not contain at least $70 \%$ of the appropriate nuclei also were excluded from analysis.

\section{Results}

\section{Lesions}

Cerebellar kainic acid lesions. Iontophoretic application of kainic acid produced successful lesions of the interpositus nucleus in $75 \%$ of the animals. It was difficult to detect the lesion 1 day after injection but by 7 days there was marked loss of neurons in the rostral 2/3rds of the interpositus nucleus (Fig. 1). There was occasional spread to the ipsilateral dentate or fastigial nuclei and there was some damage to the cerebellar cortex along the pipette track. There was no damage to distant neuronal structures including thalamus, diencephalon, hippocampus, pyriform cortex and olfactory bulb (Olney and de Gubareff 1978).

Decortication. The cerebral cortical ablations and undercuttings invariably involved motor and sensory cortex. There was frequent extension into the cingu- 


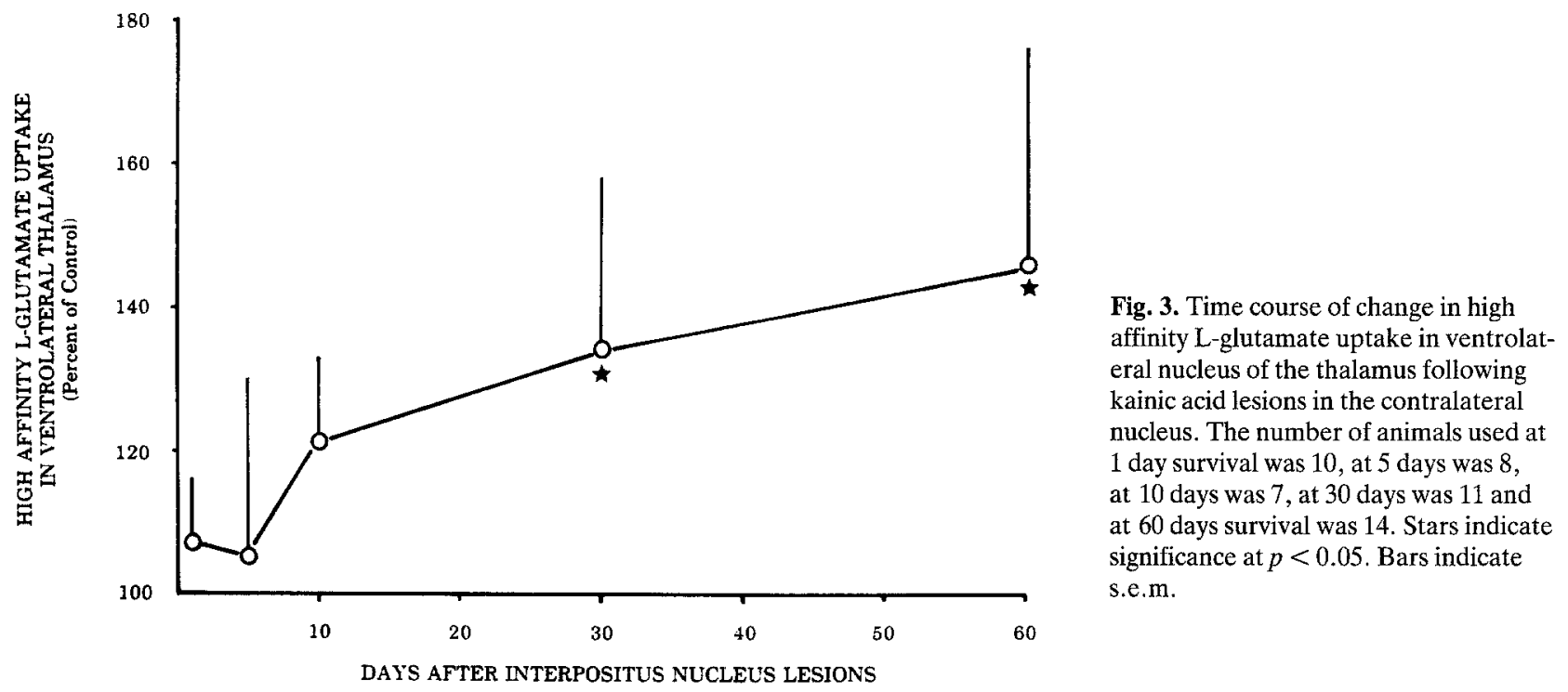

late cortex medially and to the dorsal bank of the rhinal sulcus laterally. The most rostral and occipital regions were spared. The lesions were bilaterally symmetric in extent (Fig. 2).

\section{Fink-Heimer degeneration studies}

The changes in corticorubral and corticothalamic fiber termination pattern following destruction of the interpositus fiber projection to these nuclei was studied by: a) mapping the interpositus projection and termination pattern (unilateral interpositus nucleus lesions); b) mapping the cortical projection and termination pattern (unilateral decortication); and c) mapping reactive changes (initial unilateral interpositus lesion followed 30 days later by bilateral decortication). In the last paradigm the rubral and thalamic nuclei on the side with intact interpositus input served as controls.

Preliminary studies suggested that degenerating interpositus fiber material disappeared after 30 days survival. Sacrifice 5 days after the final lesion of either the interpositus nucleus or cerebral cortex was found to be the optimum time for visualizing degenerating terminals in rubral and thalamic nuclei.

Interpositus nucleus lesions. Following lesions of the interpositus nucleus, degenerating fibers and terminals were found in accord with those previously described (Cajal 1911; Gwyn and Flumerfelt 1974; Caughell and Flumerfelt 1977). A tract of degenerating fibers could be seen which left the interpositus nucleus, travelled in the brachium conjunctivum, decussated and passed through the contralateral red nucleus where large degenerating terminals were present throughout the caudal and medial portions of the nucleus. The degenerating fibers passed rostrally with fibers of the medial lemniscus and ended in the most rostral and dorsal portions of the ventrolateral nucleus of the thalamus where dense fields of degenerating terminals were observed.

Decortication. Following decortication, ipsilateral degenerating terminals could be found mainly in the ventral and lateral portions of the rostral red nucleus (Fig. 2d), and throughout the ventrolateral thalamic nucleus (Fig. 2b), as described previously (Gwyn and Flumerfelt 1974; Brown 1974).

Serial lesions. In animals receiving an initial interpositus nucleus lesion and a subsequent decortication 30 days later, the distribution of degenerating cortical fibers in the thalamus was significant for a marked increase in density of degenerating fibers in the rostral portion of the ventrolateral nucleus (compare Fig. 2a with Fig. 2b). This was distinguishable on a blind review and was statistically significant (sign test, $p=0.05$ ). There was also a change in the distribution of degenerating terminals in the caudal red nucleus (Fig. 2f). After an initial interpositus lesion followed by a cortical lesion 30 days later, there were degenerating terminals throughout the entire extent of the red nucleus but primarily in the caudal region.

\section{Glutamate uptake}

Time course. The time course of changes in high affinity uptake of glutamate in the ventrolateral 
Table 1. Glutamate uptake in the brain stem of the rat

\begin{tabular}{|c|c|c|c|c|}
\hline Day & $\mathrm{N}$ & $\begin{array}{l}\text { Control side }{ }^{\mathrm{a}} \\
{ }^{3} \mathrm{H}-\mathrm{GLU} \mathrm{nmol} / \mathrm{mg} \text { protein } / 5 \mathrm{~min} \\
(\text { mean } \pm \mathrm{sem} \text { ) }\end{array}$ & $\begin{array}{l}\text { Average difference } \\
\text { Deafferented-control } \\
\text { side }\end{array}$ & $\begin{array}{l}\text { Average difference } \\
\text { Deafferented side as } \\
\text { percent of control }\end{array}$ \\
\hline \multicolumn{5}{|c|}{ A. Uptake from ventrolateral thalamus after unilateral interpositus lesion } \\
\hline 1 & 10 & $0.309 \pm 0.058$ & 0.039 & 107 \\
\hline 30 & 11 & $0.476 \pm 0.065$ & 0.088 & $134^{\mathrm{b}}$ \\
\hline 60 & 14 & $0.419 \pm 0.070$ & 0.148 & $146^{\mathrm{b}}$ \\
\hline \multicolumn{5}{|c|}{ B. Uptake from red nucleus after unilateral interpositus lesion } \\
\hline 1 & 5 & $0.117 \pm 0.039$ & -0.017 & 86 \\
\hline 60 & 10 & $0.087 \pm 0.011$ & 0.030 & $134^{b}$ \\
\hline \multicolumn{5}{|c|}{ C. Uptake from ventrolateral thalamus after bilateral decortication followed by unilateral interpositus lesion 30 days later } \\
\hline 30 & 8 & $0.530 \pm 0.039$ & 0.052 & 108 \\
\hline
\end{tabular}

$\mathrm{N}$ Number of animals in each experiment

${ }^{\text {a }}$ Glutamate uptake values represent measurements at glutamate concentration $1 \times 10^{-8} \mathrm{M}$

b Indicates statistical significance at $p<0.05$

thalamic nucleus after contralateral interpositus nucleus lesions was studied. The data from animals allowed to survive $1,5,10,30$, and 60 days are plotted in Fig. 3 and listed in Table 1 (part A). Uptake was not different on the two sides 1 day following a lesion, but by 10 days there was an average increase of $21 \%$ in the contralateral ventrolateral nucleus. By 30 days the average uptake had increased by $34 \%$, and by 60 days by $46 \%$ compared to the control side. The average differences were statistically significant at 30 and 60 days (analysis of variance, $p<0.05$ ). Measurements in the red nucleus were made at 1 and 60 day survival periods (Table 1 , part B). Initially, there was a slight but statistically insignificant decrease in glutamate uptake 1 day after lesions in the contralateral interpositus nucleus. At 60 days, however, there was a $34 \%$ increase in uptake following the interpositus lesion which was significant (paired t-test, $p<0.05$ ).

Saturation curves. To determine the saturation curve for glutamate uptake in the ventrolateral nucleus, 3 groups of 4 animals each received unilateral interpositus lesions. After 30 days survival, the ventrolateral nuclei from each side were pooled and assayed for ${ }^{3} \mathrm{H}$-glutamate uptake at 4 concentrations between $10^{-8}$ and $10^{-6} \mathrm{M}$. The affinity of the uptake sites on the lesioned side $\left(\mathrm{K}_{\mathrm{T}}=7.59 \pm 4.70 \times 10^{-7} \mathrm{M}\right)$ compared to the control side $\left(\mathrm{K}_{\mathrm{T}}=9.46 \pm 6.3 \times\right.$ $10^{-7} \mathrm{M}$ ) were similar. The maximal number of transport sites was higher on the lesioned side $(11.7 \pm 4.0$ $\mathrm{nmol} / \mathrm{mg}$ protein $/ 5 \mathrm{~min}$ ) compared to that of the control side $(6.7 \pm 1.0 \mathrm{nmol} / \mathrm{mg}$ protein $/ 5 \mathrm{~min})$. This increase was not statistically significant; however, the results suggest that the uptake changes observed in the time course experiments reflect primarily changes in the number of uptake sites.

Dependence upon cortical fibers. We determined if the enhanced glutamate uptake seen after interpositus lesions was dependent upon intact corticofugal fibers. Eight animals received bilateral decortication followed 5 days later by unilateral interpositus nucleus lesions. After a 35 day survival period they were sacrificed and glutamic acid uptake was measured in the ventrolateral thalamus. In these animals, there was no statistically significant difference between the two sides in glutamate uptake (Table 1, part C). These data support the notion that the integrity of corticofugal fibers to the ventrolateral thalamus are neccessary for the changes in glutamate uptake observed after interpositus nucleus lesions.

Specificity of uptake. The high affinity uptake of GABA was measured at $1 \times 10^{-8} \mathrm{M}$ using a similar technique as described for glutamate to determine if the difference in glutamate uptake described above was part of a nonspecific increase in amino acid uptake. Seven animals with interpositus lesions were assayed after 30 days survival. There was no statistically significant difference (paired t-test) in GABA uptake in the deafferented ventrolateral nucleus $(5.51 \pm 1.04 \mathrm{pmol} / \mathrm{mg}$ protein $/ 5 \mathrm{~min})$ compared to the intact side $(4.59 \pm 0.87 \mathrm{pmol} / \mathrm{mg}$ protein $/ 5 \mathrm{~min})$.

\section{Discussion}

Synaptogenesis in response to the loss of fiber terminals may be one mechanism of compensation in 
adult animals. Anatomical, electrophysiological and biochemical data supporting synaptic rearrangement have been presented for a number of sites (Stewart 1982, for review). We have concentrated on the model developed by Tsukahara and coworkers (Tsukahara 1978) which hypothesizes collateral sprouting of cortical terminals in the red nucleus after loss of fibers from the interpositus nucleus. In the present study we describe anatomical evidence (increased terminal density) and biochemical evidence (increased neurotransmitter activity) for an increase in cortical fiber influence in both the red nucleus and ventrolateral thalamic nucleus following lesions of the interpositus nucleus.

The Fink-Heimer degeneration studies agree with previous findings in the cat where there is evidence for increased numbers of corticorubral fibers and terminals following lesions of the interpositus nucleus (Nakamura et al. 1974; Murakami et al. 1982). In addition to increased numbers of degenerating products we found a change in the distribution of cortical fiber terminals with a spread to more caudal and medial regions of the red nucleus (Fig. 2e). It is not clear whether this is an entirely new field of distribution, from fibers whose terminals had previously ended only on rostrally located rubral neurons, or a marked proliferation of the rare cortical terminals observed in the caudal regions in control animals (Fig. 2f).

We have shown a similar change in the density of degenerating terminals in the ventrolateral thalamic nucleus following loss of the input from the interpositus nucleus. Although the electrophysiological changes documented in the red nucleus have not been studied in the thalamus, the principal afferent connections from the cerebellum and cerebral cortex are similar in both nuclei. Our anatomical and glutamate uptake data combined with the amino acid uptake findings of Nieoullon and Dusticier (1981) and Nieoullon et al. (1984) give strong support for including the thalamus as a site of reactive synaptogenesis.

We and others have used the technique of measuring high affinity uptake of glutamate in the synaptosomal fraction as a reliable marker of glutamatergic terminals (Young et al. 1986 for review). Although the uptake system measured is shared by glutamate and aspartate, measurement of glutamate levels provides evidence in the rat and cat for selective changes in glutamate alone, suggesting that the uptake data presented in this report reflect changes in glutamatergic terminals (Bromberg et al. 1981; Young et al. 1981).

The changes in glutamate uptake following lesions in the interpositus nucleus are similar for both the red nucleus and ventrolateral nucleus. Although our data from the ventrolateral nucleus were only statistically significant at 30 and 60 day survival times, the time course curve is clearly monotonic. The mean value of glutamate uptake was increasing 60 days after interpositus lesions and the peak of the time course is not known. The time to maximal change determined by Tsukahara from intracellular electrophysiologic studies in the red nucleus of the cat was shorter (about 10 days). Bromberg and Gilman (1978) studied extracellular multiunit rubral activity in a similar model. The time course of return of multiunit activity, which they felt reflected reactive cortical synaptogenesis, occurred over 30 to 60 days. They argued that intracellular recording might be sensitive to only the initial new proximal cortical terminals. Thus the glutamate uptake and multiunit activity studies support a longer time course of reactive synaptogenesis.

The changes in glutamate uptake were more apparent in the ventrolateral thalamic nucleus than in the red nucleus. The amount of tissue that could be obtained from the red nucleus by our techniques was at the threshold for measurement of uptake and it was difficult to reliably position the punch for maximal rubral tissue without also including contaminating tissue. This was less of a problem in the ventrolateral thalamic nucleus. The resultant poorer signal-tonoise ratio made it more difficult to show changes in the red nucleus.

The saturation curve for glutamate uptake in the ventrolateral thalamic nucleus suggests a change in the number of uptake sites only. This finding is the reverse of that found by Nieoullon et al. (1984) in similar studies in the cat. Although a possible explanation for this discrepancy is a species difference, further experiments" will be needed to verify such a possibility. The fact that the number and not the affinity of sites is changed in our study is in keeping with the increase in corticorubral terminal numbers seen anatomically.

In summary, we present anatomical and neuropharmological data to support the concept of reactive synaptogenesis in the red nucleus and ventrolateral thalamic nucleus. The increases in glutamate neurotransmitter activity, as reflected by their increased high affinity uptake, lends support to the ideas that the new cortical terminals are functional (Bromberg and Gilman 1984).

Acknowledgements. This work was supported in part by United Cerebral Palsy Educational and Research Foundation grant R-301-78 and USPHS grant NS 19613. 


\section{References}

Bromberg MB, Gilman S (1978) Changes in rubral multiunit activity after lesions in the interpositus nucleus in the cat. Brain Res 152: 353-357

Bromberg MB, Penney JB, Stephenson BS, Young AB (1981) Evidence for glutamate as the neurotransmitter of corticothalamic and corticorubral pathways. Brain Res 215: 369-374

Brown LT (1974) Corticorubral projections in the rat. J Comp Neurol 154: 149-168

Cajal RS (1911) Histologie du système nerveux de l'homme et des vertébrés, Vol II. Maloine, Paris

Caughell KA, Flumerfelt BA (1977) The organization of the cerebellorubral projection: An experimental study in the rat. J Comp Neurol 176: 295-306

Fink RP, Heimer L (1967) Two methods for selective impregnation of degenerating axons and their synaptic endings in the central nervous system. Brain Res 4: 369-374

Gwyn DG, Flumerfelt BA (1974) A comparison of the distribution of cortical and cerebellar afferents in the red nucleus of the rat. Brain Res 69: 130-135

Kerkerian L, Nieoullon A, Dusticier N (1982) Brain glutamate uptake: regional distribution study from sensorimotor areas in the cat. Neurochem Int 4: 275-281

Kerkerian L, Nieoullon A, Dusticier N (1984) Topographical changes in high affinity glutamate uptake from the cat red nucleus, substantia nigra, thalamus and caudate nucleus following lesions of sensorimotor cortical areas. Exp Neurol 81: $598--612$

McGeer PL, McGeer EG, Hattori T (1978) Kainic acid as a tool in neurobiology. In: McGeer EG, Olney JW, McGeer PL (eds) Kainic acid as a tool in neurobiology. Raven Press, New York, pp 123-138

Murakami F, Katsumaru H, Saito K, Tsukahara N (1982) A quantitative study of synaptic reorganization in red nucleus neurons after lesion of the nucleus interpositus of the cat: an electron microscopic study involving intracellular injection of horseradish peroxidase. Brain Res 242: 41-53

Nakamura Y, Mizuno N (1971) An electron microscopic study of the interpositorubral connections in the cat and rabbit. Brain Res 35: 283-286
Nakamura Y, Mizuno N, Konishi A, Sato M (1974) Synaptic reorganization of the red nucleus after chronic deafferentation from cerebellorubral fibers: an electron microscope study in the cat. Brain Res 82: 298-301

Nieoullon A, Dusticier N (1981) Increased glutamate decarboxylase activity in the red nucleus of the adult cat after cerebellar lesions. Brain Res 224: 129-139

Nieoullon A, Kerkerian L, Dusticier N (1984) High affinity glutamate uptake in the red nucleus and ventrolateral thalamus after lesion of the cerebellum in the adult cat: biochemical evidence for functional changes in the deafferented structures. Exp Brain Res 55: 409-419

Olney JW, de Gubareff T (1978) Extreme sensitivity of olfactory cortical neurons to kainic acid toxicity. In: McGeer EG, Olney JW, McGeer PL (eds) Kainic acid as a tool in neurobiology. Raven Press, New York, pp 201-217

Pamel G, Stephenson B, Penney JB, Bromberg MB, Young AB (1981) Biochemical evidence for sprouting of corticothalamic fibers after interpositus nucleus lesions in rat. Neurology 31 : 150

Steward O (1982) Assessing the functional significance of lesioninduced neuronal plasticity. Int Rev Neurobiol 23: 197-254

Tsukahara N, Hultborn H, Murakami F, Fujito Y (1975) Electrophysiological study of formation of new synapses and collateral sprouting in red nucleus after partial denervation. J Neurophysiol 38: 1359-1374

Tsukahara N (1978) Synaptic plasticity in the red nucleus. In: Cotman CW (ed) Neuronal plasticity. Raven Press, New York, pp 113-130

Young AB, Bromberg MB, Penney JB (1981) Decreased glutamate uptake in subcortical areas deafferented by sensorimotor cortical ablation in the cat. J Neurosci 1: 241-249

Young AB, Penney JB, Dauth GW, Bromberg MB, Gilman S (1983) Glutamate or aspartate as a possible neurotransmitter of cerebral corticofugal fibers in the monkey. Neurology 33 : 1513-1516

Young AB, Frey KA, Agranoff BW (1986) Receptor assays: in vitro and in vivo. In: Phelps M, Mazziotta J, Schelbert $\mathrm{H}$ (eds) Positron emission tomography and autoradiography: principles and applications for the brain and heart. Raven Press, New York, pp 73-111

Received September 5, 1986 / Accepted September 7, 1987 